\title{
Propagating Health Insurance: A Formative Evaluation of Broadcast Media Programs Sponsored by National Health Insurance Scheme (NHIS) in Selected Northern States of Nigeria (2007-2012)
}

Hamajoda A $^{*}$

National Institute for Legislative Studies (NILS), Nigeria

\begin{abstract}
This paper is based on a survey carried out in 2013 to assess the formative potential of broadcast programs designed to propagate the need for health insurance among citizens in six Northern States of Nigeria from 2007 to 2012. Like many insurance schemes across the world, the National Health Insurance Scheme (NHIS) envisaged contributors to pay into common pools to enable health care providers to attend to the contributors in medical needs by drawing from the pool of funds. This risk-sharing scheme is fathomed to improve resource mobilization and improve equity especially in underdeveloped situations like in Nigeria, where poverty has increased child and maternal mortality and reduced access to good medical care. NHIS media strategy focuses on convincing citizens on the benefits of enrolling in the schemes. The strategy also assists stakeholders to disseminate knowledge of the scheme to potential enrollees, and to ensure mass participation of citizens by ensuring adequate news coverage of NHIS events.
\end{abstract}

Keywords: Health Insurance Scheme; Health communication; Evaluation of media campaigns; Media strategy; Formative evaluation

\section{Introduction}

The National Health Insurance Scheme (NHIS) is established to ensure the availability of good healthcare to citizens in Nigeria. It is established under Act 35 of 1999 by the Federal Government of Nigeria to improve the health of all Nigerians at an affordable cost. Although the NHIS kick-started in 2005, the idea has been muted since 1962 and have been revisited in 1984, 1989 until 1997 when the scheme was formally launched.

Like many insurance schemes across the world, the NHIS envisaged contributors to pay into common pools to enable health care providers to attend to the contributors in medical needs by drawing from the pool of funds. This risk-sharing scheme is fathomed to improve resource mobilization and improve equity especially in underdeveloped situations like in Nigeria where poverty has increased child and maternal mortality and reduced access to good medical care. Sekhri and Saredoff in discussing the potentials of health insurance for developing countries draw attention to the immediate protective benefits of reducing financial burdens on households and the frequent harassment of 'large out-of-pocket expenditures' bedeviling underdeveloped countries [1].

Following a national clamor for health policy, a National Health Act 2014 was signed "to provide a framework for the regulation, development and management of a national health system and set standards for rendering health services in the federation, and other matters connected therewith, 2014". According to Jimoh, the act guarantees a basic health care for citizens to be funded by an annual grant of not less than one per cent of Nigeria's Consolidated Revenue Fund and grants by international donor partners and funds from any other sources [2]. Out of such collected funds, $50 \%$ shall be used for the provision of basic minimum package of health services to citizens, in eligible primary/or secondary health care facilities through the National Health Insurance Scheme (NHIS). Sekhri and Saredoff point to how central funding is to covering the poor in health insurance in order to avoid the prospect of health insurance that is narrowed to the middle class [1]. Although the NHIS since 2007 did embark on a national advocacy programs to mobilize communities in Nigeria mostly involving formal launchings of the scheme in several communities in Nigeria, data collected by this survey indicates that the perception of the scheme as purely designed for government workers still persists.

Central to the mandate of NHIS in improving the health care of citizens at an affordable cost are two categories of health organizations, HMOs and HCPs. Health Maintenance Organizations under NHIS carry out the central function of managed healthcare by financing and ensuring the delivery of health services especially referrals of registered clients, to secondary and tertiary medical levels. In doing so, HMOs shift the financial risk for healthcare from clients and sponsors, to service providers. Most cases that involve referrals to specialists must be approved by them. On the other hand, the Health Care Providers (HCPs) are the first service providers to registered clients. They are made of clinics, hospitals, and other health centers that attend to the client mostly through their outpatient departments. They form over $70 \%$ of client health attendance.

In order to ensure that every Nigerian has access to good health care services, the National Health Insurance Scheme has developed various products to cover different segments of the society and these are:
i. Formal Sector Social Health Insurance Programme
ii. Urban Self-employed Social Health Insurance Programme

*Corresponding author: Hamajoda A, National Institute for Legislative Studies (NILS), Nigeria, Tel: 2348023493382; E-mail: aminufhamajoda@yahoo.com

Received October 05, 2015; Accepted October 23, 2015; Published October 31, 2015

Citation: Hamajoda A (2015) Propagating Health Insurance: A Formative Evaluation of Broadcast Media Programs Sponsored by National Health Insurance Scheme (NHIS) in Selected Northern States of Nigeria (2007-2012). J Mass Communicat Journalism 5: 280. doi:10.4172/2165-7912.1000280

Copyright: (C) 2015 Hamajoda A. This is an open-access article distributed unde the terms of the Creative Commons Attribution License, which permits unrestricted use, distribution, and reproduction in any medium, provided the original author and source are credited. 
Citation: Hamajoda A (2015) Propagating Health Insurance: A Formative Evaluation of Broadcast Media Programs Sponsored by National Health Insurance Scheme (NHIS) in Selected Northern States of Nigeria (2007-2012). J Mass Communicat Journalism 5: 280. doi:10.4172/21657912.1000280

Page 2 of 7

iii. Rural Community Social Health Insurance Programme

iv. Children Under-Five Social Health Insurance Programme

v. Permanently Disabled Persons Social Health Insurance Programme

\section{vi. Prison Inmates Social Health Insurance Programme}

vii. Tertiary Institutions and Voluntary Participants Social Health Insurance Programme

viii. Armed Forces, Police and other Uniformed Services

\section{Role of Broadcast Media}

According to Rimal and Lapinski, Health communication is seen to have relevance for virtually every aspect of health and well-being, including disease prevention, health promotion and quality of life" [3]. Health insurance being new to Nigerians necessitated the need for advocacy and communication strategies to create awareness among the various stakeholders and target groups. NHIS media strategy focuses on convincing citizens on the benefits of enrolling in its floated schemes. The strategy also assists stakeholders to disseminate knowledge of the scheme to potential enrollees, and to ensure mass participation of citizens by ensuring adequate news coverage of NHIS events.

Communication projects that aim at social change in Africa by using mass media face several problems that primarily center on infrastructure, dearth of authentic data especially in media usage, lack of content analysis of programs to ascertain their desirability, and extremely low audience survey that leaves both institutions, content producers and even the broadcasters themselves in the wild. One of the consequences of these shortcomings is that most social projects hardly care to start their communication projects with a comprehensive detail blueprint that consist of goals, messages, audience, channels, content, monitoring and evaluation. Sixsmith attest that the consequences of these obstacles affect the efficient evaluation of health-related media campaigns and encourage the exclusion of base-line studies and communication components in most projects [4].

From 2007 to 2012, without a baseline study, NHIS floated a series of radio jingles, TV commercials, TV dramas and documentaries that were aired in the six geo-political zones of the country. In Northern Nigeria, the area this study is concerned with, the following programs were aired:

- 60 seconds radio jingle on FRCN (Federal Radio Corporation of Nigeria)

- 60 seconds radio jingle on local stations

- 60 seconds TV commercial on Network News

- 60 seconds TV commercial on Local stations

- 30 minutes Drama on Local Stations

- 30 minutes TV talk show on Local Stations

- 30 minutes TV documentary on NTA Network

- 30 minutes Radio talk show on Radio Kaduna

\section{Overview of NHIS Sponsored Broadcast Programs}

The programs consist of jingles, talk-shows, documentaries and drama Tables 1a-d. One of the most important reasons for the formative survey was to find out if funding of TV and radio programs as communication interventions are worthwhile. Similarly NHIS wanted to know if communication interventions were to be expanded

\begin{tabular}{|l|l|}
\hline PROGRAM TITLE & BetaBodi \\
\hline DURATION & 30 minutes \\
\hline FORMAT & TV Talk show \\
\cline { 2 - 2 } & Involving an interview by a host with an NHIS official in front of an audience. Each episode focus on an aspect of the \\
& NHIS in terms of products and stakeholders. The production is interspersed with jingles, interviews and even drama \\
\hline
\end{tabular}

Table 1a: Shows program title Beta Bodi.

\begin{tabular}{|l|l|}
\hline PROGRAM TITLE & Easy Access to Health Care \\
\hline DURATION & 30 minutes \\
\hline FORMAT & $\begin{array}{l}\text { TV Documentary (NETWORK) } \\
\text { Featuring detail explanations of NHIS products using endorsement interviews, discussion of challenges and } \\
\text { achievements, interlude jingles, interviews, information desk. All NTA local Stations were expected to hook-up for } \\
\text { the program. }\end{array}$ \\
\hline
\end{tabular}

Table 1b: Shows program title Easy Access to Health Care.

\begin{tabular}{|c|c|}
\hline PROGRAM TITLE & Gaskiya Dokin Karfe \\
\hline DURATION & 30 minutes \\
\hline FORMAT & $\begin{array}{l}\text { Radio Feature } \\
\text { Aired on Radio Kaduna. A Hausa News Feature program covering NHIS occasions across the country using a host } \\
\text { who use reports, interviews, product explanations, and endorsement interviews that educate people about NHIS } \\
\text { products. Radio Kaduna is received in most Northern states. }\end{array}$ \\
\hline \multicolumn{2}{|c|}{ Table 1c: Shows program title Gaskiya Dokin Karfe. } \\
\hline PROGRAM TITLE & Radio Jingle \\
\hline DURATION & 60 seconds \\
\hline FORMAT & $\begin{array}{l}\text { Radio Jingle } \\
\text { In Pidgin English using folk drama to advocate the advantage of equity contribution in healthcare in rural poverty } \\
\text { situation. }\end{array}$ \\
\hline
\end{tabular}

Table 1d: Shows program title Radio Jingle. 
Citation: Hamajoda A (2015) Propagating Health Insurance: A Formative Evaluation of Broadcast Media Programs Sponsored by National Health Insurance Scheme (NHIS) in Selected Northern States of Nigeria (2007-2012). J Mass Communicat Journalism 5: 280. doi:10.4172/21657912.1000280

Page 3 of 7

or scaled down. At the end of the study, NHIS wanted to know if to replicate its TV and Radio programs and to ascertain if the contents were justified in relation to the key messages NHIS will like its clients and stakeholders to be aware of.

The survey also sought to find out which channel people prefer in accessing information on their health and other wellbeing requirements. Among the targets the survey decided to find out the actual popularity of the various channels to help NHIS to target its messages more effectively. The survey also significantly sought to find out how the various media products designed to raise awareness have reached the audience and how target groups could be reached effectively.

The peculiarity of radio and television transmission mainly lies in their unidirectionality. Whereby institutions assume that whatever is broadcasted is watched or listened to by target audience, they may come to realise that this may not be so. Health-geared media intervention programs like jingles, talk-shows, drama etc ultimately aim at empowering people to take control of improving their health. Central to assessing the potential impact of intervention communication products, is the accurate understanding of the main purpose of the intervention scheme. In the case of NHIS, it is clearly enshrined in A-Z of NHIS Manual. The role of the media is stipulated as to "help the agency in the areas of Advocacy, Sensitization and Mass mobilisation of the public".

So in general the communication intervention strategy that was studied had aimed at the general education and information of both stakeholders and the general public about the products of NHIS and the essence of health insurance in ensuring equity in health access and the development of health facilities.

\section{The Survey Indicators and Questions}

The indicators that were studied are:

- Percentage of audience who listened/viewed/understand the messages.

- $\quad$ Percentage of people who express knowledge, attitude and belief consistent with the message of the programs.

- Percentage of people who have acquired the products or skills recommended by the communication.

In that context, the survey endevoured to find out if citizens were aware of NHIS products and the choice they can make and if the floated media products had in any way attributed to this awareness. In the absence of a baseline survey prior to impact evalution, Freimuth discuss how formative studies can help provide useful data and challenges that 'can help planners understand how to effectively tailor mass communication strategies to the receiver characteristics of homogenous segments of the population' [5]. Impact on people and stakeholders therefore were measured to ensure first, people have knowledge about health insurance, second, are motivated through awareness about the equity scheme of NHIS, and third have the skills to negotiate their health and that of their families.

The formative indicators for NHIS media strategy under the study were:

- Increased knowledge and awareness of the importance of health insurance in ensuring health care access and the expansion of health care facilities.

- Awareness of NHIS products and how they work.

\section{- Enrolment into one of NHIS products}

The survey therefore measured the awareness of NHIS products and the essence of health care insurance. About $50 \%$ of potential enrollees should exhibit general awareness of the importance of health insurance and the key product that affects them.

\section{Methodology}

Prior to this study, there was not any baseline study that was conducted before the airing of programs. In a way therefore this survey is both post-interventionist and a formative evaluation. The questionnaire prepared (Appendix A) sought to find out popular broadcast stations, types of programming that interest potential enrollees, their preferred source of health news, their viewing and listening habits and their general attitude to health insurance. The survey used the KABP measuring technique which relies on several questions to reveal knowledge, behavior and practice of target audience. This focus always fairly evaluate the impact of broadcast messages be they jingles, drama, talk shows, documentaries and others. The KABP method is more pertinent because controlled groups were not created prior to the contract award to media companies by NHIS.

Key population among the stakeholders had included:

- Leaders of Rural Community Based Insurance

- $\quad$ Self Employed Urban Dwellers

- Public Private Sector Employers

- Disable Persons

- $\quad$ Key Operators of NHIS Schemes in HCP

- Members of Professions and Uniformed Services

- $\quad$ Students in Tertiary Institutions.

Six states were selected, Adamawa, Gombe, Katsina, Kaduna, Niger, and Kwara. A list of broadcast stations (Radio and TV) in each state was prepared with the recognition that some stations transverse boundaries. In that regard for each state, the segment on broadcast stations was varied according to the existing stations in each state including receivership of trans-border stations (e.g Radio Kaduna), network stations and cable stations. A structured questionnaire (Appendix A) was prepared in English. It was translated into Hausa and Yoruba by field officers while conducting the survey as required. The questionnaire was apportioned to not less than 10 field officers in each state according to the list of key population and stakeholder's segmentation. The questionnaire targeted selected population in each target state (Civil Servants, Self-employed, Urban Dwellers, Traders, Store Keepers, Parent Workers, Disabled Persons, Uniformed Men, and Tertiary Students). Essentially the questionnaire (Appendix A) endeavored to find out from respondents 10 vital information relevant to the above discussed indicators necessary for further communication planning by NHIS.

a) The kind of information gadgets possessed by responders.

b) The broadcast stations they tune to in their respective states.

c) The types of programming that interest them.

d) Responders' knowledge of the number of products floated by

NHIS.

e) The date of enrolment to any NHIS product if any. 
Citation: Hamajoda A (2015) Propagating Health Insurance: A Formative Evaluation of Broadcast Media Programs Sponsored by National Health Insurance Scheme (NHIS) in Selected Northern States of Nigeria (2007-2012). J Mass Communicat Journalism 5: 280. doi:10.4172/21657912.1000280

Page 4 of 7

f) Source of knowledge about NHIS.

g) Which among the floated broadcast programs responders ever watched or listened.

h) Preferred primary medium for health information.

i) Attitude to health insurance.

At the end of the survey, the following number of filled questionnaires were returned, Gombe (100), Katsina (101), Kaduna (151), Adamawa (145), Niger (110), Kwara (101).

\section{Results and Discussions}

Central to communication data analysis is the determination of the key questions and indicators in the communication strategy. This being done efficiently and refined and consolidated during implementation and collection of feedbacks, made it easy to develop a structure for analysis.

After the collection of data an intuitive understanding of emerging themes and concerns developed. These emerging themes and concerns guided the organization of collected data, so the analysis stage was an exercise in relating such themes to already galvanized indicators and questions. Essentially the process of data analysis was done using EPI Info7 and Microsoft Excel. A baseline database was created in EPI Info7 as a receptacle for the raw data collected, and later each frequency analysis (single or combined) was exported to MS Excel for charting.

Health insurance is undoubtedly one of the most cogent ways of unshackling the health sector from low government funding and reliance on donors. However communicating the equity strategies of the NHIS scheme and the logical ways they can help reduce health burden on both the rich and the poor require formidable communication and advocacy plans. Questionnaire administrators during the survey were incessantly besieged with questions from the public who want to know more. This is not surprising as people are driven to access information that can potentially improve their health and NHIS should exploit this universal need. The phenomenon is particularly noted by Skuse and Butler who discuss how people generally in underdeveloped countries value information on their livelihoods and health [6]. But this enthusiasm can also be tainted by certain myths, like the view that health insurance is 'elitist' or a 'government workers scheme' as noted by Sekhri and Saredoff [1]. Surveyors encountered this view overwhelmingly on the field, posing a potential hindrance to the promotion of health insurance across the various states of Nigerian.

Several related studies that assess level of awareness and effectiveness of NHIS in selected cities and working groups were carried out. Owu for instance studied the level of awareness among federal workers in Kaduna metropolis, which he found to be of 'high level', nevertheless it would have been pertinent to compare his findings to the awareness levels of state and local workers, private sector workers and self-employed workers, which this study found to be at low levels [7]. In fact, Chubike confirmed the low level awareness among state civil servants in two cities Enugu and Abakaliki [8]. According to Chibuke findings, 'civil servants working with Ebonyi and Enugu State Governments do not know much about the NHIS' [8]. Sanusi and Awe also note the limitation of coverage among all strata of the society. All the studies therefore call for greater enlightenment and education regarding health insurance [9].

\section{Information gadgets possessed by responders}

Surprising FM radio ranks higher than TV sets in respondents information gadgets possession (Figure 1), the highest being radio sets. Close to $60 \%$ of respondents listen to radio always making the medium predictably the most important in health communication. As Figure 2 shows, Local TV unfortunately rank far below cable TV in terms of viewing explaining why the drama and talk-shows floated by NHIS fared badly among other reasons.

\section{Sources of knowledge about NHIS}

The survey found that majority of registered people heard about NHIS from their employers not the media before registration as was planned.

As Figure 3 shows, $48 \%$ of registered respondents indicated that

\section{Percent}

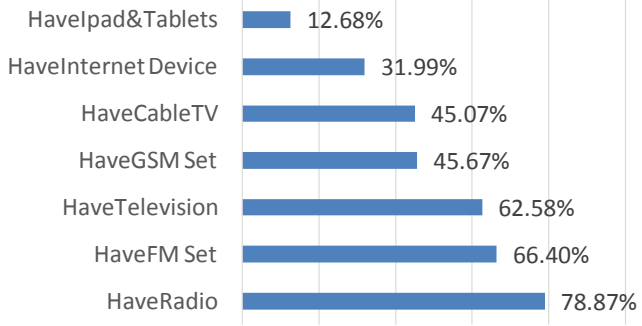

$0.00 \% 20.00 \% 40.00 \% 60.00 \% 80.00 \% 100.00 \%$

Figure 1: Information Gadgets possessed by Respondents.

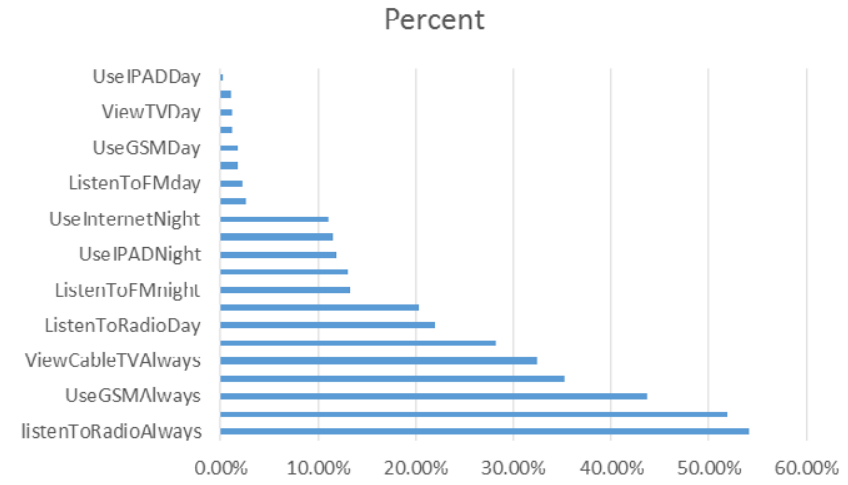

Figure 2: Channels Usage by Respondents.

\section{Information about NHIS before registration}

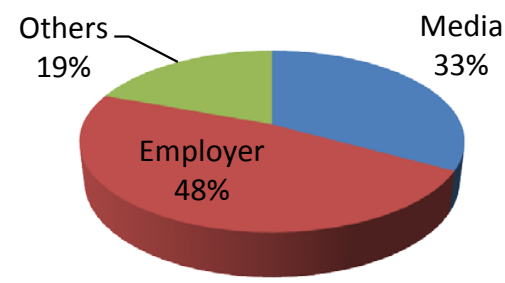

Figure 3: Source of Information about NHIS to Registered Responders. 
Citation: Hamajoda A (2015) Propagating Health Insurance: A Formative Evaluation of Broadcast Media Programs Sponsored by National Health Insurance Scheme (NHIS) in Selected Northern States of Nigeria (2007-2012). J Mass Communicat Journalism 5: 280. doi:10.4172/21657912.1000280

Page 5 of 7

they received information about the NHIS from their work place before registration and not through the media as the campaign targeted. It means developing propagation strategies in organizations, OPDs in medical centers and pharmacies will help in communicating NHIS programs far more than the broadcast media.

\section{Knowledge of the number of products floated by NHIS}

As Figure 4 shows, most people polled especially the self-employed, traders and artisans cited lack of information for their inability to register with NHIS. Lack of information constitute $50 \%$ of inability to register as compared to a total of other reasons like funds, culture, personal and other assorted reasons given by potential clients.

Majority of respondents believe that the NHIS has only one package - for the civil servants. Some state workers actually believe that only federal workers are entitled to register with the scheme. The other packages of the insurance scheme are not known at all. The NHIS information handbill carried by surveyors was a revelation and many respondents asked for a copy. It is therefore important that when revising the contents of broadcast programs that a way is instituted to make sure that all the insurance packages of the NHIS are propagated.

\section{Program ranking}

It appears NHIS NTA Network programs and jingles paid dividends in the sense that a total of $68 \%$ of respondents have acknowledged seeing Easy Access to Healthcare and the Network NHIS TV advert at least once.

As Figure 5 shows, rather Ineffectiveness is found in the local airtimes that were purchased. Programs like Beta Bodi and Gaskiya Dokin Karfe scored low in states where they were aired. Figures 6 and 7 below for instance, show how NHIS floated programs fared in Kaduna and Kwara States as typical examples. Only the jingles aired on FRCN Kaduna and NTA Network news and to a similar extend Gaskiya Dokin

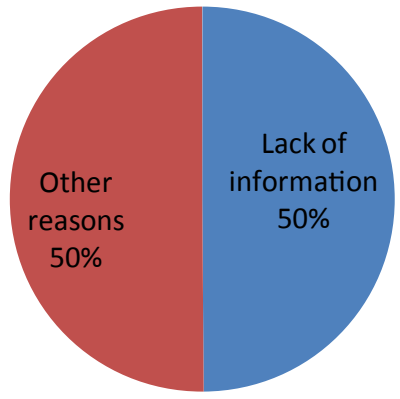

- Lack of information

Other reasons

Figure 4: Percentage of Responders who attribute lack of Information to NHIS products.

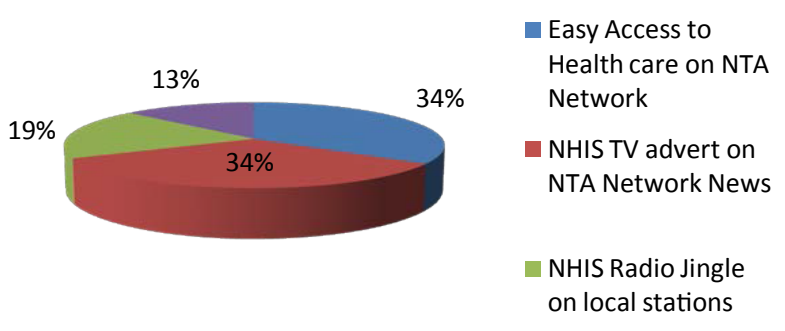

Figure 5: Effectiveness in percentage of radio and TV jingles aired.

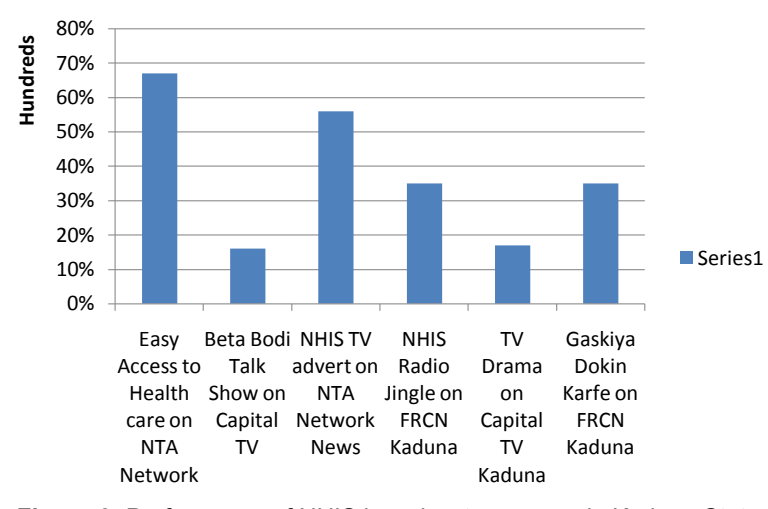

Figure 6: Performance of NHIS broadcast programs in Kaduna State.

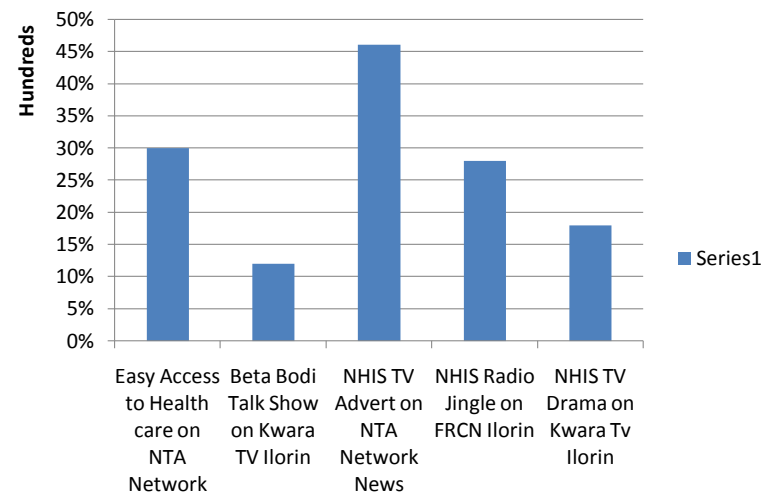

Figure 7: Performance of NHIS broadcast programs in Kwara State.

Karfe, a Hausa feature scored above $35 \%$ as Figure 6 shows. Jingles also fared better than programs in Kwara State Figure 7 far better than the talk-show and drama aired.

\section{Broadcast stations tuned to by responders in their respective states}

The dearth of popularity index of local stations might have prevented a correct decision to target popular stations in each state for the airing of programs and jingles. Getting a reliable popularity index of broadcast stations and programs in Nigeria, and indeed Africa in general, is one of the major obstacles in communication planning that target broadcasting stations. Southwood describes the state of audience broadcast research in Africa as "blissful ignorance" and "gut feel" in the absence of continuous audience research that makes media planning difficult [10]. This survey found that NHIS programs targeting local stations are not aired on popular channels. In all the states surveyed, popular stations surprising are not those that were targeted by NHIS. This problem is more so with radio stations where popular FMs were not selected at all for the broadcast of radio jingles. In Kaduna for instance Brila Radio and Supreme FM are the most popular radio channels according to Figure 8.

\section{Preferred medium for health information}

$39 \%$ of people who were polled rely on radio for health information, followed by newspapers (Figure 9). Anastsui emphasizes how radio programming can substitute lack of formal education and infrastructural underdevelopment to reach rural areas with practical 
Citation: Hamajoda A (2015) Propagating Health Insurance: A Formative Evaluation of Broadcast Media Programs Sponsored by National Health Insurance Scheme (NHIS) in Selected Northern States of Nigeria (2007-2012). J Mass Communicat Journalism 5: 280. doi:10.4172/21657912.1000280

Page 6 of 7

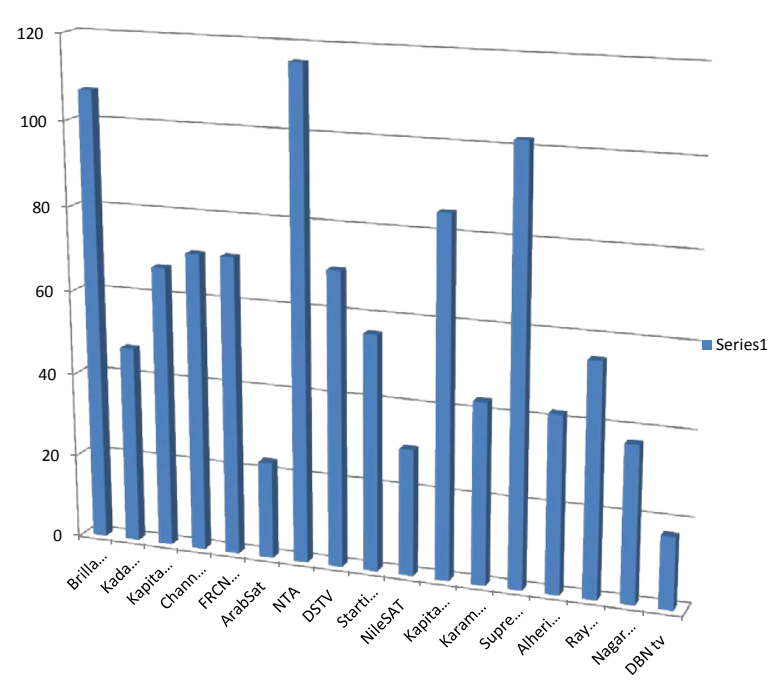

Figure 8: Popularity of Broadcast Stations According to Responders in Kaduna State.

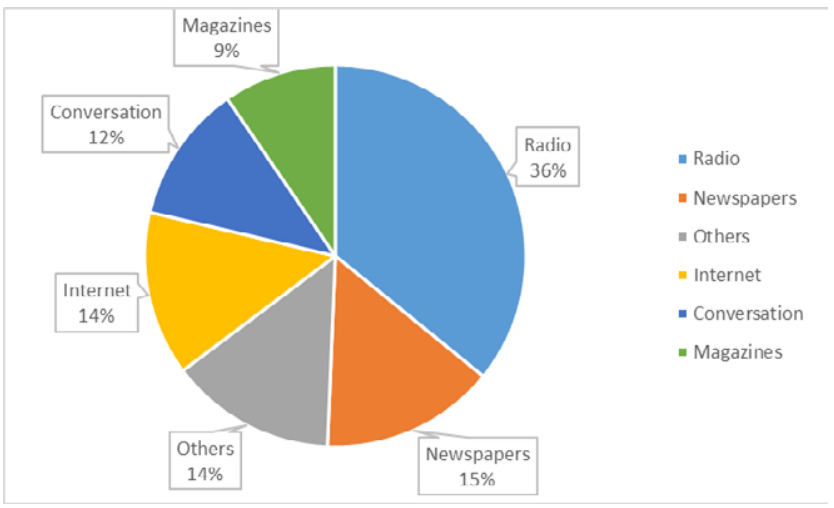

Figure 9: Preferred Medium for Health Information.

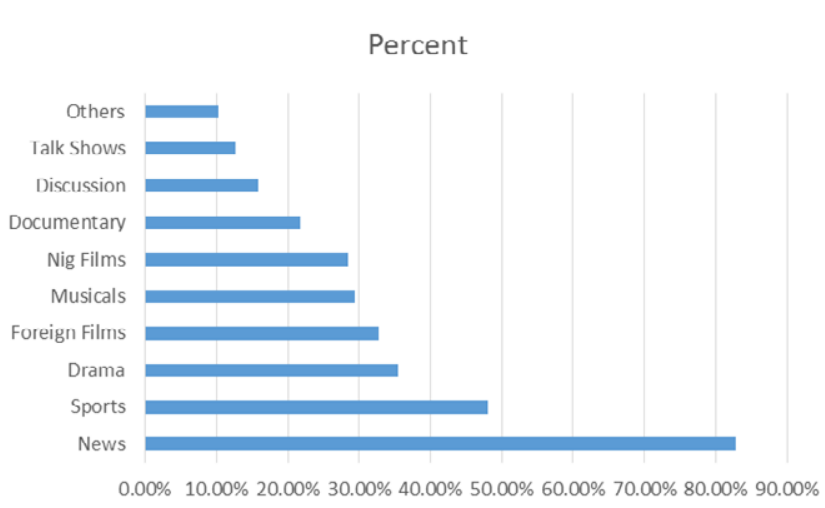

Figure 10: Ranking of Program Types that interest respondents.

information about health and other existential needs [11]. It is pertinent therefore to develop more radio programs in propagating the logic of health insurance. But to do this, more detail surveys must be conducted to ascertain popular local stations and the kind of programs that attract audience in all states of Nigeria. Stations must be studied in terms of reach, popularity and programming.

\section{Types of broadcast programming that interest respondents}

News and sports rank as the most liked broadcast programs as Figure 10 shows. Probably this explains the reason for the comparable impact made by NHIS NTA Network packages which are aired before or during Network news when citizens tune in at least once to know what is happening in the nation. In redesigning broadcast programs, a strategy should be worked out to ensure comprehensive news coverage that include explanatory materials on the logic of health insurance and the type of packages available for all segments of the population. Lots of endorsement packages are required.

\section{Conclusions and Recommendations}

The evaluation project started could aid a full fledge communication planning, implementation, monitoring and evaluation scheme. Communication is currently facing the challenge of expanded mediality, where choice of channels is more critical than developing messages. Unfortunately media and audience research is very limited in Africa as pointed out earlier. It is pertinent therefore to expand the population of the survey focusing on more research into media channels and popularly programming to form a baseline. This is crucial because currently NHIS has achieved over $70 \%$ of public sector registration and achieving the same level for the community-based and other packages will be herculean.

Community-based projects require a formidable communication plan. Adhoc programming and acceptance of proposals from outside media companies should be discarded. All content production should be done within a framework that should be developed by NHIS based on this and more surveys.

This survey reaffirms the need for comprehensive communication plan for all public projects that require media campaigns in Nigeria. Such plans should focus on situation analysis, identification of goals, detail design of messages for channels, audience appraisal, discussion of channels, effective timeline, monitoring and evaluation strategies, and all other best practices. This study has shown that more than any agency, NHIS require intensive communication in this stage of its development.

\section{References}

1. Sekhri N, Saredoff W (2004) Private Health Insurance: Implications for Developing Countries. World Health Organization, Geneva

2. Jimoh A (2014) Understanding The National Health Act.

3. Rimal RN, Lapinski MK (2009) Why Health Communication is Important in Public Health. Bulletin of the World Health Organization 87: 247-247.

4. Sixsmith J, Fox KA, Doyle P, Barry MM (2014) A Literature Review on Health Communication Campaign Evaluation with Regard to the Prevention and Control of Communicable Disease in Europe. ECDC, Stockholm.

5. Freimuth V, Cole G, Kirby S (2001) Issues in Evaluating Mass Media-Based Health Communication Campaigns.

6. Skuse A (2004) Radio Broadcasting for Health: An Issue Paper. Information and Communication for Development, DFID, London.

7. Owu E, Ifatimehen O, Shaka S (2014) Assessment of the Level of Awareness of the Effectiveness of National Health Insurance Scheme among Workers in Kaduna Metropolis Nigeria. J of Education and Policy Review V 6: 43-51.

8. Chubike NE (2013) Evaluation of National Health Insurance Scheme (NHIS) Awareness by Civil Servants in Enugu and Abakaliki. International Journal of Medicine and Medical Sciences 5: 356-358.

9. Sanusi RA, Awe AT (2009) An Assessment of Awareness Level of Nationa Health Insurance Scheme among Health Consumers in Oyo State Nigeria. The Social Science 4: 143-148. 
Citation: Hamajoda A (2015) Propagating Health Insurance: A Formative Evaluation of Broadcast Media Programs Sponsored by National Health Insurance Scheme (NHIS) in Selected Northern States of Nigeria (2007-2012). J Mass Communicat Journalism 5: 280. doi:10.4172/21657912.1000280

Page 7 of 7

10. Southwood R (2012) Broadcast audiences - Africa's blissful ignorance holds back industry development in most of the continent.
11. Anatsui TC (2008) The Role of Broadcasting in Health Communication for National Development in Nigeria. JORIND 12. 